\title{
THE TRENDS HIGH-CONTRAST IMAGING SURVEY. III. A FAINT WHITE DWARF COMPANION ORBITING HD 114174
}

\author{
Justin R. Crepp ${ }^{1}$, John Asher Johnson ${ }^{2}$, Andrew W. Howard ${ }^{3}$, Geoffrey W. Marcy ${ }^{4}$, \\ Alexandros Gianninas ${ }^{5}$, Mukremin Kilic ${ }^{5}$, and Jason T. Wright 6,7 \\ ${ }^{1}$ Department of Physics, University of Notre Dame, 225 Nieuwland Science Hall, Notre Dame, IN 46556, USA; jcrepp@ nd.edu \\ 2 Department of Planetary Science, California Institute of Technology, 1200 E. California Blvd., Pasadena, CA 91125 , USA \\ ${ }^{3}$ Institute for Astronomy, University of Hawaii, 2680 Woodlawn Drive, Honolulu, HI 96822, USA \\ ${ }^{4}$ Department of Astronomy, University of California, Berkeley, CA 94720, USA \\ ${ }^{5}$ Department of Physics and Astronomy, University of Oklahoma, Norman, OK 73019, USA \\ ${ }^{6}$ Department of Astronomy \& Astrophysics, The Pennsylvania State University, University Park, PA 16802, USA \\ ${ }^{7}$ Center for Exoplanets and Habitable Worlds, The Pennsylvania State University, University Park, PA 16802, USA \\ Received 2013 May 2; accepted 2013 June 24; published 2013 August 7
}

\begin{abstract}
The nearby Sun-like star HD 114174 exhibits a strong and persistent Doppler acceleration indicating the presence of an unseen distant companion. We have acquired high-contrast imaging observations of this star using NIRC2 at Keck and report the direct detection of the body responsible for causing the "trend." HD 114174 B has a projected separation of $692 \pm 9$ mas $\left(18.1 \mathrm{AU}\right.$ ) and is $10.75 \pm 0.12 \mathrm{mag}$ (contrast of $5 \times 10^{-5}$ ) fainter than its host in the $K$-band, requiring aggressive point-spread function subtraction to identify. Our astrometric time baseline of $1.4 \mathrm{yr}$ demonstrates physical association through common proper motion. We find that the companion has absolute magnitude, $M_{J}=13.97 \pm 0.11$, and colors, $J-K=0.12 \pm 0.16 \mathrm{mag}$. These characteristics are consistent with an $\approx \mathrm{T} 3$ dwarf, initially leading us to believe that HD $114174 \mathrm{~B}$ was a substellar object. However, a dynamical analysis that combines radial velocity measurements with available imaging data indicates a minimum mass of $0.260 \pm 0.010 M_{\odot}$. We conclude that HD $114174 \mathrm{~B}$ must be a white dwarf. Assuming a hydrogen-rich composition, atmospheric and evolutionary model fits yield an effective temperature $T_{\text {eff }}=8200 \pm 4000 \mathrm{~K}$, surface gravity $\log g=8.90 \pm 0.02$, and cooling age of $t_{c} \approx 3.4 \mathrm{Gyr}$, which is consistent with the $4.7_{-2.6}^{+2.3} \mathrm{Gyr}$ host star isochronal age estimate. HD $114174 \mathrm{~B}$ is a benchmark object located only $26.14 \pm 0.37 \mathrm{pc}$ from the Sun. It may be studied at a level of detail comparable to Sirius and Procyon, and used to understand the link between the mass of white dwarf remnants with that of their progenitors.
\end{abstract}

Key words: astrometry - stars: individual (HD 114174) - techniques: high angular resolution - techniques: radial velocities - white dwarfs

Online-only material: color figures

\section{INTRODUCTION}

The TRENDS (TaRgetting bENchmark-objects with Doppler Spectroscopy) high-contrast imaging program uses precise radial velocity $(\mathrm{RV})$ measurements obtained over a long time baseline to identify promising targets for follow-up observations using adaptive optics (AO; Crepp et al. 2012b, 2013). In addition to diffraction-limited imaging, TRENDS also uses coronagraphy, point-spread function (PSF) subtraction, and $\approx 1 \mathrm{hr}$ integration times to achieve deep contrast at near-infrared wavelengths (Marois et al. 2006; Lafrenière et al. 2007; Crepp et al. 2010). The primary goal of TRENDS is to image and characterize benchmark M-dwarfs, brown dwarfs, and (ultimately) extrasolar planets, using a combination of complementary substellar companion detection techniques, in order to calibrate theoretical evolutionary models and theoretical spectral models of cool dwarf atmospheres.

Precise Doppler measurements for TRENDS are obtained at Keck Observatory using the HIgh Resolution Echelle Spectrometer (HIRES; Vogt et al. 1994) as part of the California Planet Search program (e.g., Howard et al. 2010). Targets with the longest RV time baselines have complementary data taken at Lick Observatory using the $0.6 \mathrm{~m}$ Coude Auxiliary Telescope and Shane $3 \mathrm{~m}$ Telescope with the Hamilton spectrometer (Vogt 1987). High-contrast imaging follow-up observations are conducted at Keck using NIRC2 (instrument PI: Keith Matthews) with the Keck II AO system (Wizinowich et al. 2000), and at Palomar using Project 1640 with the PALM-3000 high-order AO system (Hinkley et al. 2011).

Crepp et al. (2012b, hereafter TRENDS I) reported the discovery of three benchmark high-mass ratio binaries, HD 53665, HD 68017, and HD 71881, each companion having an age and metallicity estimate inferred from the solar-type primary. Arguably the most interesting object of the three, HD $68017 \mathrm{~B}$, has a RV time baseline of $15 \mathrm{yr}$ and shows significant orbit curvature. With a projected separation of only $13.0 \mathrm{AU}$, it will be possible to measure a precise dynamical mass in the next several years. The model-dependent mass of HD $68017 \mathrm{~B}$ from relative photometry is $m_{\text {model }}=168 \pm 21 M_{J}\left(0.16 \pm 0.02 M_{\odot}\right)$.

Crepp et al. (2013, TRENDS II) reported the direct detection of an $m_{\text {model }}=0.52 \pm 0.04 M_{\odot}$ tertiary companion orbiting the 84 day period, single-line spectroscopic binary HD 8375 AB. With a separation of $0 ! 3$, HD $8375 \mathrm{C}$ has a brightness comparable to residual scattered starlight from the inner binary pair prior to PSF subtraction. The HD 8375 system represents one of many hierarchical triples in the solar neighborhood that are known to exist, through statistical analysis of multiplicity fractions for stellar systems within $d<10 \mathrm{pc}$, but so often evade direct detection (Tokovinin 2004).

In this paper (TRENDS III), we report the discovery of a faint object orbiting the nearby, $d=26.14 \pm 0.37 \mathrm{pc}$ (van Leeuwen 2007), G5 star HD 114174 (LHS 2681, HIP 


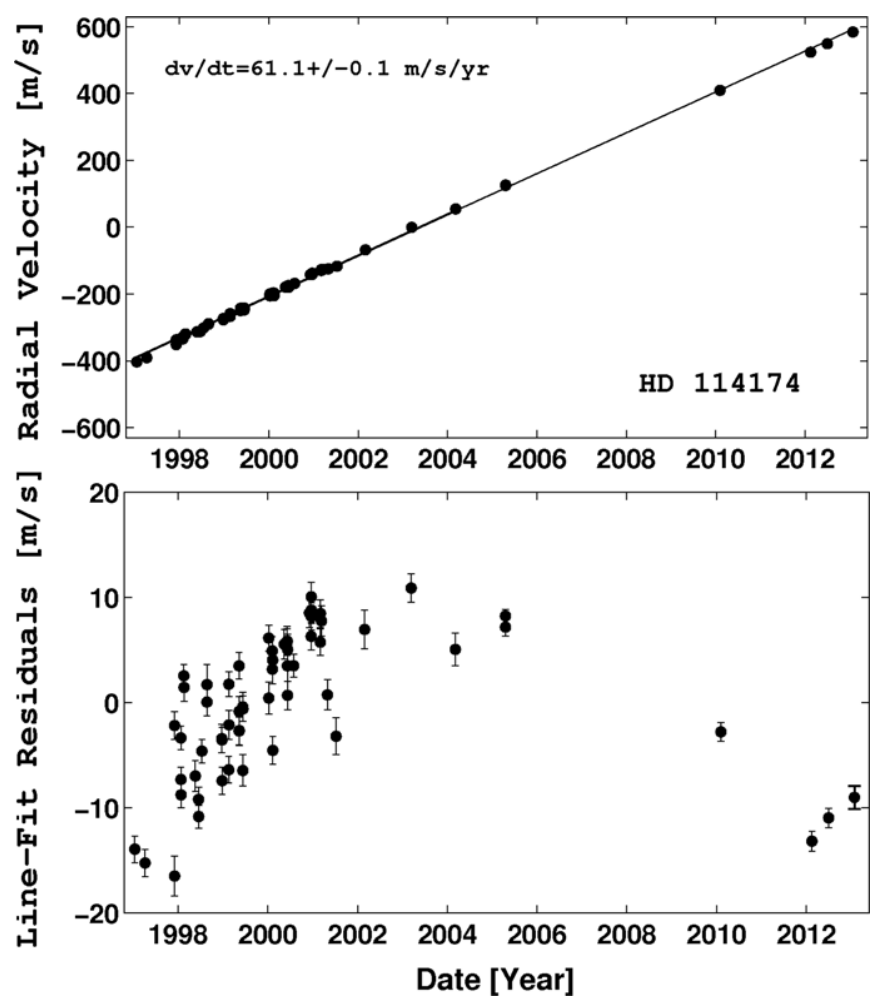

Figure 1. Relative radial velocity measurements of HD 114174 from the years 1997 through 2013. Top: a long-term Doppler acceleration of $61.1 \pm$ $0.1 \mathrm{~m} \mathrm{~s}^{-1} \mathrm{yr}^{-1}$ implies the presence of a companion with a long period. Bottom: a linear fit model results in large residuals indicating systemic curvature (change in the acceleration). Thus, it will soon be possible to calculate the threedimensional orbit and dynamical mass of HD $114174 \mathrm{~B}$.

64150, GJ 9429). The companion, HD 114174 B, cannot be seen without significant on-source integration time and PSF subtraction. It has a projected separation $(0.69=18.1 \mathrm{AU})$ comparable to the semimajor axis $(a=18.3 \mathrm{AU})$ of the brown dwarf HR 7672 B, which recently yielded a dynamical mass with a fractional error of only 4\% (Crepp et al. 2012a). Initially characterized as a substellar object based on its brightness and colors, we find that HD 114174 B must instead be a white dwarf, in order to establish a self-consistent interpretation with long-term Doppler RV measurements.

Few "Sirius-like" systems, i.e., directly imaged white dwarfs around solar-type stars, are presently known-approximately one dozen within 20 pc (Holberg 2009). Our serendipitous discovery of a compact object orbiting HD 114174 thus constitutes a new member of a small collection of nearby, directly detected benchmark white dwarfs for which we can determine physical properties independent of spectro-photometric measurements. Forthcoming measurements will ultimately lead to tight dynamical constraints on its orbit and mass. More than $16 \mathrm{yr}$ of RV measurements are already in hand.

\section{OBSERVATIONS}

\subsection{High-resolution Stellar Spectroscopy}

\subsubsection{Doppler Measurements}

We obtained RV data of HD 114174 using HIRES at Keck. The standard iodine cell referencing method was used to calibrate instrument drifts and measure precise Doppler shifts (Marcy \& Butler 1992). Our Doppler observations began on UT 1997 January 14. Several years of measurements revealed that the star exhibits a (mostly) linear acceleration indicative of a distant companion. Figure 1 shows Doppler observations taken over an 16.0 yr timeframe. RV values, epochs, and measurement uncertainties are listed in Table 1.

Treating the Doppler trend as purely linear and using a Markov Chain Monte Carlo (MCMC) fitting procedure, we find an acceleration of $61.1 \pm 0.1 \mathrm{~m} \mathrm{~s}^{-1} \mathrm{yr}^{-1}$. This analysis includes a differential offset between measurements obtained before and after the summer 2004 HIRES detector upgrade, which we treat as a free parameter. Closer inspection of the data shows that the RV slope is slowly changing with time. For instance, the bottom panel of Figure 1 displays RV residuals once the measurements are subtracted from a linear model. Continued Doppler monitoring will allow us to place tight constraints on the companion orbit and dynamical mass, as soon as the direct imaging astrometry shows curvature. By performing a joint RVastrometry analysis using presently available data, we derive a lower-limit for the mass of HD 114174 B (Section 5.1) and determine a range of plausible orbital periods (Section 5.4).

\subsubsection{Stellar Properties}

Stellar template spectra, taken with the iodine gas cell removed from the optical path, were analyzed using the LTE spectral synthesis code Spectroscopy Made Easy (SME) described in Valenti \& Piskunov (1996) and Valenti \& Fischer (2005). SME provides an estimate of the stellar effective temperature $\left(T_{\text {eff }}\right)$, surface gravity $(\log g)$, metallicity $([\mathrm{Fe} / \mathrm{H}])$, and projected rotational velocity $(v \sin i)$. The estimated physical properties of HD 114174 derived from spectral fitting are shown in Table 2.

HD 114174 is listed in the SIMBAD database as a G5(IV) subgiant. However, its location with respect to the mean Hipparcos main-sequence (Wright 2005) and spectroscopic $\log g=4.51 \pm 0.06$ value indicate the star is a main-sequence dwarf. Placing HD 114174 on a Hertzsprung-Russell diagram, we estimate a mass of $1.05 \pm 0.05 M_{\odot}$ and age of $4.7_{-2.6}^{+2.3} \mathrm{Gyr}$ by comparing spectroscopic parameters to Yonsei-Yale theoretical isochrone tracks. Despite its close distance, HD 114174 would rarely, if ever, make typical high-contrast imaging target lists, which nominally only select stars younger than $\approx 1$ Gyr (Crepp \& Johnson 2011).

\subsection{High-contrast Imaging}

We acquired first-epoch high-contrast images of HD 114174 on UT 2011 February 22 using NIRC2 and the Keck II AO system (Wizinowich et al. 2000). The bright $\left(K_{s}=5.20\right.$; Skrutskie et al. 2006) star was placed behind the 300 mas diameter coronagraphic spot. We used the angular differential imaging (ADI) technique to discriminate between residual scattered starlight and companions through PSF subtraction (Marois et al. 2006).

Raw frames were processed by flat-fielding the array, replacing bad pixels with interpolated values, and high-pass Fourier filtering. The latter step reduces the AO halo and thermal background (pedestal) while accentuating quasi-static speckles, which aids with image alignment. Frames were then multiplied by a gray-scale intensity mask (having contiguous transmission values between 0 and 1 ) to prevent the coronagraph from biasing image alignment. We used the Locally Optimized Combination of Images (LOCI) algorithm to increase effective contrast levels (Lafrenière et al. 2007). The subtracted frames were then rotated to the standard east-north orientation using the parallactic angle to combine images and stack light from any off-axis sources. 
Table 1 HD 114174 Keck HIRES RV Measurements

\begin{tabular}{|c|c|c|}
\hline HJD-2,450,000 & $\begin{array}{c}\mathrm{RV} \\
\left(\mathrm{m} \mathrm{s}^{-1}\right)\end{array}$ & $\begin{array}{c}\text { Uncertainty } \\
\left(\mathrm{m} \mathrm{s}^{-1}\right)\end{array}$ \\
\hline 463.1438 & -403.39 & 1.27 \\
\hline 545.9798 & -390.77 & 1.29 \\
\hline 787.1381 & -351.45 & 1.89 \\
\hline 787.1439 & -337.14 & 1.33 \\
\hline 838.0563 & -333.70 & 1.17 \\
\hline 838.1109 & -329.74 & 1.10 \\
\hline 839.1258 & -334.99 & 1.20 \\
\hline 862.0168 & -319.81 & 1.06 \\
\hline 862.9814 & -320.76 & 1.30 \\
\hline 955.8422 & -313.57 & 1.45 \\
\hline 981.7798 & -311.44 & 1.18 \\
\hline 982.7957 & -312.89 & 1.09 \\
\hline 1009.8359 & -302.12 & 1.13 \\
\hline 1050.7552 & -288.92 & 1.92 \\
\hline 1050.7572 & -290.58 & 1.29 \\
\hline 1171.1521 & -273.81 & 1.35 \\
\hline 1172.1624 & -273.77 & 1.22 \\
\hline 1173.1611 & -277.47 & 1.31 \\
\hline 1227.0404 & -267.37 & 1.28 \\
\hline 1228.0764 & -259.05 & 1.17 \\
\hline 1228.9999 & -262.77 & 1.37 \\
\hline 1310.8726 & -249.58 & 1.33 \\
\hline 1311.8551 & -243.23 & 1.27 \\
\hline 1312.8933 & -247.37 & 1.42 \\
\hline 1313.9960 & -249.02 & 1.40 \\
\hline 1340.8177 & -242.25 & 1.39 \\
\hline 1341.8676 & -248.12 & 1.49 \\
\hline 1342.8364 & -242.09 & 1.21 \\
\hline 1553.1269 & -205.71 & 1.55 \\
\hline 1553.1298 & -200.00 & 1.22 \\
\hline 1582.0826 & -196.36 & 1.34 \\
\hline 1583.0577 & -197.94 & 1.41 \\
\hline 1585.0085 & -196.73 & 1.20 \\
\hline 1585.0130 & -196.74 & 1.19 \\
\hline 1586.0218 & -205.15 & 1.34 \\
\hline 1679.8938 & -179.28 & 1.42 \\
\hline 1702.9103 & -175.20 & 1.28 \\
\hline 1703.8065 & -174.94 & 1.35 \\
\hline 1704.8793 & -177.12 & 1.49 \\
\hline 1705.8784 & -179.77 & 1.35 \\
\hline 1706.8757 & -175.24 & 1.43 \\
\hline 1755.7542 & -168.56 & 1.11 \\
\hline 1884.1599 & -141.95 & 1.41 \\
\hline 1898.1674 & -141.82 & 1.28 \\
\hline 1899.1728 & -137.89 & 1.37 \\
\hline 1900.1678 & -139.61 & 1.30 \\
\hline 1901.1771 & -138.81 & 1.27 \\
\hline 1972.1057 & -129.95 & 1.26 \\
\hline 1973.1304 & -127.04 & 1.33 \\
\hline 1982.1034 & -126.24 & 1.43 \\
\hline 2030.9064 & -125.05 & 1.43 \\
\hline 2101.8222 & -117.06 & 1.75 \\
\hline 2334.1177 & -67.85 & 1.84 \\
\hline 2712.0408 & -0.36 & 1.37 \\
\hline 3071.9391 & 54.34 & 1.56 \\
\hline \multicolumn{3}{|c|}{ RV Offset } \\
\hline 3480.0014 & 130.10 & 0.86 \\
\hline 3480.0026 & 131.17 & 0.62 \\
\hline 5232.1301 & 414.79 & 0.91 \\
\hline 5973.1039 & 529.01 & 0.95 \\
\hline 6109.7547 & 554.22 & 0.92 \\
\hline 6319.0020 & 584.50 & 1.10 \\
\hline
\end{tabular}

Notes. A horizontal line indicates the division between HIRES (detector) preupgrade and post-upgrade data points, requiring a differential offset for analysis. Uncertainties correspond to photon-noise alone.
Table 2

HD 114174 Properties

\begin{tabular}{|c|c|}
\hline \multicolumn{2}{|c|}{ HD 114174 Properties } \\
\hline Right ascension [J2000] & 130851.02 \\
\hline Declination [J2000] & +051226.06 \\
\hline$B$ & 7.47 \\
\hline$V$ & 6.80 \\
\hline$J_{2 M A S S}$ & $5.613 \pm 0.026$ \\
\hline$K_{s}$ & $5.202 \pm 0.023$ \\
\hline$J_{\mathrm{MKO}}$ & $5.584 \pm 0.027$ \\
\hline$K_{\text {MKO }}$ & $5.189 \pm 0.024$ \\
\hline$d(\mathrm{pc})$ & $26.14 \pm 0.37$ \\
\hline Proper motion ( mas $\mathrm{yr}^{-1}$ ) & $\begin{array}{r}84.72 \pm 0.59 \mathrm{E} \\
-670.11 \pm 0.47 \mathrm{~N} \\
\end{array}$ \\
\hline \multicolumn{2}{|c|}{ Host Star } \\
\hline Mass $\left(M_{\odot}\right)$ & $1.05 \pm 0.05$ \\
\hline $\operatorname{Radius}\left(R_{\odot}\right)$ & 1.06 \\
\hline Luminosity $\left(L_{\odot}\right)$ & 1.13 \\
\hline Age (Gyr) & $4.7_{-2.6}^{+2.3}$ \\
\hline$[\mathrm{Fe} / \mathrm{H}]$ & $0.07 \pm 0.03$ \\
\hline $\log g\left(\mathrm{~cm} \mathrm{~s}^{-2}\right)$ & $4.51 \pm 0.06$ \\
\hline$T_{\text {eff }}(K)$ & $5781 \pm 44$ \\
\hline Spectral type & G5 IV-V \\
\hline$v \sin i\left(\mathrm{~km} \mathrm{~s}^{-1}\right)$ & $1.8 \pm 0.5$ \\
\hline \multicolumn{2}{|c|}{ Companion $^{a}$} \\
\hline$\Delta J$ & $10.48 \pm 0.11$ \\
\hline$\Delta K$ & $10.75 \pm 0.12$ \\
\hline$\Delta L^{\prime}$ & $>9.84$ \\
\hline$J$ & $16.06 \pm 0.11$ \\
\hline$K$ & $15.94 \pm 0.12$ \\
\hline$L$ & $>14.99$ \\
\hline$M_{J}$ & $13.97 \pm 0.11$ \\
\hline$M_{K}$ & $13.85 \pm 0.12$ \\
\hline$M_{L^{\prime}}$ & $>12.90$ \\
\hline$T_{\text {eff }}(K)$ & $8200 \pm 4000$ \\
\hline $\log g\left(\mathrm{~cm} \mathrm{~s}^{-2}\right)$ & $8.90 \pm 0.02$ \\
\hline$t_{c}(\mathrm{Gyr})$ & $\approx 3.4$ \\
\hline$m_{\mathrm{dyn}}\left(M_{\odot}\right)$ & $>0.260 \pm 0.010$ \\
\hline$m_{\text {model }}\left(M_{\odot}\right)$ & $1.15 \pm 0.01$ \\
\hline
\end{tabular}

Notes. (Top) Coordinates, apparent magnitudes, distance, and proper motion of HD 114174 from the SIMBAD database. Magnitudes are from Two Micron All Sky Survey (2MASS; Skrutskie et al. 2006). Filter conversions from Carpenter (2001) have been applied to estimate stellar MKO values. The parallax-based distance is from Hipparcos measurements using the refined data reduction of van Leeuwen (2007). (Middle) Host star physical properties are estimated from SME using HIRES template spectra as well as theoretical isochrones (Valenti \& Fischer 2005). (Bottom) Companion magnitude difference, apparent magnitude, absolute magnitude, and companion physical properties, such as the estimated cooling age $\left(t_{c}\right)$, mass constraint (lower-limit) from dynamics $\left(m_{\mathrm{dyn}}\right)$, and mass $\left(m_{\text {model }}\right)$ from photometry using white dwarf evolutionary models assuming a pure hydrogen atmosphere.

Upon processing the full 2011 February data set, we noted a faint candidate companion located $\approx 0$ !'7 to the south in the final reduced image. Follow-up observations were taken on UT 2012 February 2, May 29, and July 4 with the $K^{\prime}$ and $J$ filters to obtain color information and determine whether the candidate is associated with the primary. Figure 2 shows the confirmation image taken one year after the first epoch.

We also obtained a deep ADI sequence of HD 114174 with NIRC2 in the $L^{\prime}$-band on UT 2012 May 7. These observations were recorded under good conditions $(\approx 0$ '.5 seeing), at low airmass (1.03-1.05), and achieved 40.5 of field rotation, yet resulted in a non-detection, suggesting that HD $114174 \mathrm{~B}$ may 


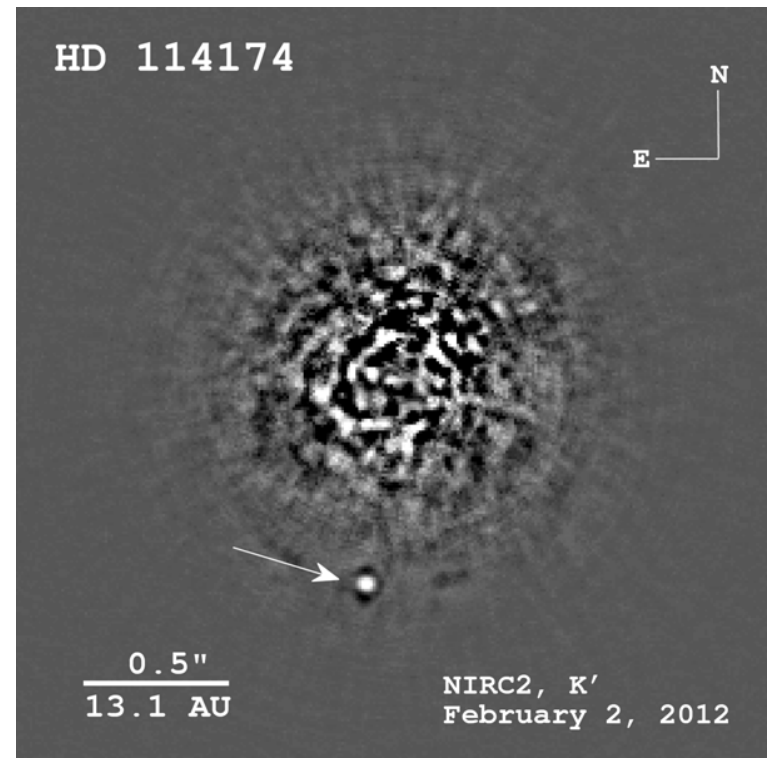

Figure 2. Confirmation image of HD 114174 B taken with NIRC2 at Keck. The $\Delta K^{\prime}=10.73 \pm 0.12$ companion cannot be seen in individual raw frames with long exposures, but is clearly visible after PSF subtraction.

have blue colors. We derive a lower-limit to the brightness difference between HD $114174 \mathrm{~B}$ and its parent star of $\Delta L^{\prime}>$ $9.84 \mathrm{mag}$. The companion was recovered at all other epochs using filters at shorter wavelengths.

\section{PHOTOMETRY}

PSF subtraction modifies the measured flux (photometry) and location (astrometry) of faint companions that are initially buried in bright speckle noise (Crepp et al. 2011; Pueyo et al. 2012). We injected synthetic companions into the NIRC2 ADI data to estimate the amount of companion flux lost as a result of using the LOCI algorithm (Soummer et al. 2012). Selfconsistent introduction of fake companions into each frame also allows us to measure the intrinsic scatter in photometric and astrometric quantities and thus compute their uncertainty.

We measured the relative brightness between HD $114174 \mathrm{~B}$ and its host star by comparing the flux from LOCI-processed images to unocculted images of the primary star, accounting for the difference in integration time. A fast-readout, subarray mode prevented HD 114174 A from saturating the array in unocculted frames. Subsequent reductions using fake companions and the same LOCI parameters were then performed to determine the (throughput) response of the data pipeline. The injected companions were placed at approximately the same angular separation as HD $114174 \mathrm{~B}$, but with azimuthal separations sufficient to prevent artificial self-subtraction (Quanz et al. 2012). Unocculted frames of the primary star informed our choice of the FWHM for fake companions.

Measurements using two different filters, $K_{\mathrm{MKO}}^{\prime}$ and $J_{\mathrm{MKO}}$, provided relative flux and color information. Optimized for the local atmospheric transmission and thermal background on Mauna Kea (Tokunaga et al. 2002), the $K_{\text {MKO }}^{\prime}$ band is our filter choice for search-mode operation. We measure flux ratios of $\Delta K_{\mathrm{MKO}}^{\prime}=10.73 \pm 0.12$ and $\Delta J_{\mathrm{MKO}}=10.48 \pm 0.11$ (Table 2). We then convert the $J_{2 \text { MASs }}$ and $K_{s}$ magnitudes of the primary star to $J_{\mathrm{MKO}}$ and $K_{\mathrm{MKO}}$ using the $J_{2 \mathrm{MASS}}-K_{s}$ color from SIMBAD and empirical transformations from Carpenter (2001).
To place the companion flux in the same filter basis, ${ }^{8}$ we downloaded high-resolution spectra of various cold dwarfs from the IRTF spectral library ${ }^{9}$ as well as the filter profiles from Keck Observatory. ${ }^{10}$ IRTF library spectra from a G5 dwarf, HD 165185, served as a fiducial to compare the colors of the companion to the G5 primary, HD 114174 A. HD 165185 and HD 114174 A have $J-K_{s}$ colors consistent to within 0.045 mag (Skrutskie et al. 2006), smaller than the 0.12 mag uncertainty in the measured companion-to-star flux difference.

Our initial interpretation of the companion's properties led us to believe it was a brown dwarf. We thus began filter conversion calculations using IRTF spectra from two cold brown dwarfs listed in Cushing et al. (2005), SDSS J125453.90-012247.4 (T2) and 2MASS J05591915-1404489 (T4.5). We first scaled the flux of the different T-dwarf spectra by a multiplicative factor to match our $\Delta K_{\mathrm{MKO}}^{\prime}$ measurement, then compared the $\Delta J_{\text {MKO }}$ model result to our $\Delta J_{\text {MKO }}$ measurement. The cooler T4.5 dwarf spectrum resulted in a significantly better fit to our measurements $\left(\Delta J_{\mathrm{MKO}}\right.$ within $\left.1 \sigma\right)$ than the T2 dwarf spectrum which was discrepant at the $5 \sigma$ level. Using the T4.5 spectrum reference, we find $\Delta K_{\mathrm{MKO}}=10.75 \pm 0.12$ (from the measured $\left.\Delta K_{\mathrm{MKO}}^{\prime}=10.73 \pm 0.12\right), K_{\mathrm{MKO}}=15.94 \pm 0.12$, and $(J-K)_{\mathrm{MKO}}=0.12 \pm 0.16$ (Table 2$)$.

Both the absolute magnitude and colors of HD $114174 \mathrm{~B}$ are consistent with a cold brown dwarf (Figure 3). However, as shown below (Sections 5.1 and 5.2), our dynamical analysis precludes this interpretation. Since the spectral energy distribution of cool white dwarfs and late-type brown dwarfs are both often dominated by collisionally induced $\mathrm{H}_{2}$ absorption (Borysow \& Frommhold 1990), and the correction factor between the $K_{\mathrm{MKO}}^{\prime}$ and $K_{\mathrm{MKO}}$ filters is small, particularly for dwarfs with neutral colors, we adopt the $\Delta K_{\mathrm{MKO}}=10.75 \pm 0.12$ value for subsequent analysis.

\section{ASTROMETRY}

Our astrometric observations consist of four epochs taken over an $1.4 \mathrm{yr}$ baseline (Table 3 ). The proper-motion of HD

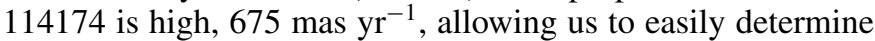
whether the companion shares the same space motion as the primary. We measured an accurate separation and position angle using the technique described in Crepp et al. (2012a). Following PSF subtraction, we fit Gaussian functions to the stellar and companion PSF to locate their centroids in each frame. The results from trials using numerous fake injected companions are averaged and uncertainty in the separation and position angle are initially taken as the measurement standard deviation. We then account for uncertainty in the plate scale and instrument orientation (Ghez et al. 2008) by propagating these errors to the final calculated position. Finally, a 5 mas uncertainty is added in quadrature to the rectilinear position of the star to account for unknown distortions introduced by the focal plane occulting mask (Q. Konopacky 2011, private communication). For companions with intensity comparable to, or fainter than, stellar quasi-static speckles using (near) Nyquist-sampled data, it is difficult to measure an astrometric position with precision much less than a single pixel (Marois et al. 2010; Crepp et al. 2011).

\footnotetext{
8 Central wavelength for the $K_{\mathrm{MKO}}^{\prime}$ and $K_{\mathrm{MKO}}$ filters are $\lambda_{K^{\prime}}=2.124 \mu \mathrm{m}$ and $\lambda_{K}=2.196 \mu \mathrm{m}$.

9 http://irtfweb.ifa.hawaii.edu/ spex/IRTF_Spectral_Library/

10 http://www2.keck.hawaii.edu/inst/nirc2old/filters.html
} 
Table 3

Summary of Astrometric Measurements

\begin{tabular}{lccccc}
\hline \hline $\begin{array}{l}\text { Date } \\
(\mathrm{UT})\end{array}$ & JD-2,450,000 & Filter & $\begin{array}{c}\rho \\
(\mathrm{mas})\end{array}$ & $\begin{array}{c}\text { Position Angle } \\
\left({ }^{\circ}\right)\end{array}$ & $\begin{array}{c}\text { Projected Separation } \\
(\text { AU) }\end{array}$ \\
\hline 2011 Feb 22 & 5615.1 & $K^{\prime}$ & $719.8 \pm 6.6$ & $171.8 \pm 0.5$ & $18.82 \pm 0.32$ \\
2012 Feb 2 & 5960.1 & $K^{\prime}$ & $701.1 \pm 5.0$ & $172.1 \pm 0.4$ & $18.33 \pm 0.29$ \\
2012 May 29 & 6076.8 & $K^{\prime}$ & $695.8 \pm 5.8$ & $170.5 \pm 0.6$ & $18.19 \pm 0.25$ \\
2012 Jul 4 & 6112.8 & $J$ & $692.1 \pm 8.7$ & $172.2 \pm 0.8$ & $18.09 \pm 0.34$ \\
\hline
\end{tabular}
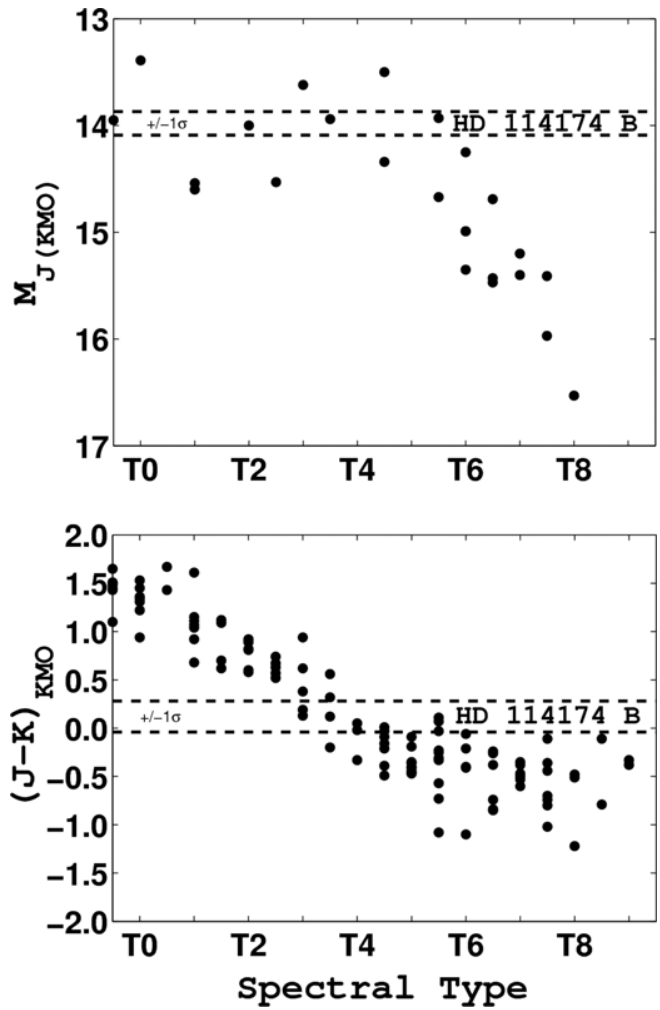

Figure 3. HD 114174 B absolute $J$-band magnitude and $J-K$ color compared to cold brown dwarfs from Leggett et al. (2010). Black data points represent spectral types derived from moderate resolution spectroscopy (http://www.gemini.edu/staff/sleggett). Our $1 \sigma$ measurement uncertainties from broadband photometry are shown as horizontal lines. HD $114174 \mathrm{~B}$ appears to be consistent with a T3.5 dwarf, but dynamical considerations indicate otherwise. Given the comparable near-infrared colors between brown dwarfs and white dwarfs, compact objects like HD $114174 \mathrm{~B}$ might be considered as a type of false-positive for high-contrast imaging surveys dedicated to searching for substellar companions.

Figure 4 shows multi-epoch astrometry measurements plotted against the expected motion of a distant background object. We find that HD $114174 \mathrm{~B}$ is clearly associated with HD $114174 \mathrm{~A}$. The position of the companion has changed by only 28 mas over an $1.4 \mathrm{yr}$ time-frame. An unrelated background source placed at infinite distance with zero proper-motion would have moved relative to the host star by 902 mas over the same timeframe. HD $114174 \mathrm{~B}$ has a projected separation of $18.09 \pm 0.34 \mathrm{AU}$ (UT 2012 July 4) that appears to be decreasing with time.

\section{COMPANION PROPERTIES}

\subsection{Mass Lower-limit from Dynamics}

A lower-limit to the companion mass may be determined from the RV acceleration and direct imaging angular separation (Torres 1999; Liu et al. 2002). Although the HD 114174 Doppler signal already shows a hint of curvature (Figure 1), we do

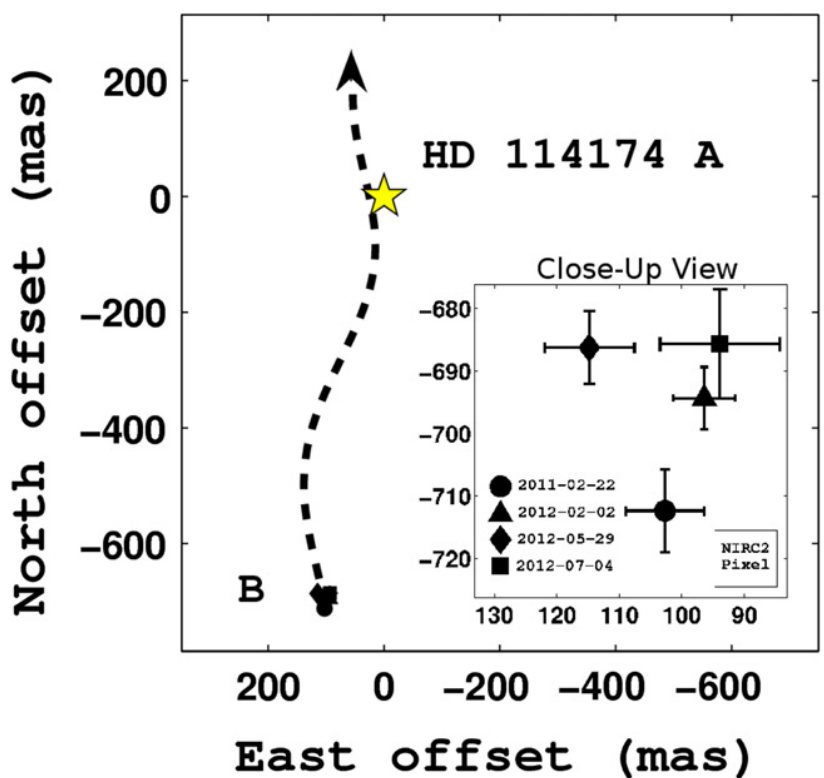

Figure 4. Astrometric measurements demonstrating that HD $114174 \mathrm{~B}$ is comoving with its host star. Location of the primary defines the coordinate system and is denoted by a star. The dashed curve shows the path that a distant background object would execute from UT 2011 February 22 through UT 2012 July 4, accounting for proper-motion and parallactic motion.

(A color version of this figure is available in the online journal.)

not compute the full range of possible masses because the astrometry has yet to show significant orbital motion, which is required to place interesting constraints on orbital elements and the companion mass (Kalas et al. 2008; Currie et al. 2011; Crepp et al. 2012a, 2012b). Instead, we evaluate the local slope of the RV time series (see also Section 5.4). We have obtained a recent Doppler RV observation (2013 January 26) to facilitate this calculation. Using an MCMC simulation to fit the instantaneous RV slope, we find a best-fitting Doppler acceleration of $57.4 \pm 0.4 \mathrm{~m} \mathrm{~s}^{-1} \mathrm{yr}^{-1}$, a value similar to that found in Section 2.1 when considering the full RV data set because curvature in the RV time-series is still subtle. This measurement translates to a minimum dynamical mass of $0.260 \pm 0.010 M_{\odot}$, thus precluding the interpretation that HD $114174 \mathrm{~B}$ is substellar, despite its low luminosity and neutral colors.

\subsection{White Dwarf Interpretation}

White dwarfs are a common outcome of the evolutionary process for low-mass stars and are thus expected to comprise a non-negligible fraction of stellar companions (Holberg et al. 1998; Breton et al. 2012). With a sample of more than 100 targets, it is therefore not surprising that the TRENDS program would discover a compact object orbiting a nearby star. Indeed, it seems the only self-consistent interpretation of our photometric and dynamical measurements is that HD $114174 \mathrm{~B}$ is a white 
dwarf companion. Such serendipitous discoveries might be considered as direct imaging false-positives when searching for exoplanets and brown dwarfs (Zurlo et al. 2013).

Despite the prevalence of white dwarfs throughout the galaxy (Oppenheimer et al. 2001), relatively few benchmark compact objects have been discovered (Muirhead et al. 2013) and only several nearby companions with precise parallax measurements, such as Sirius B and Procyon B, have been imaged directly (Liebert et al. 2005). Similar to field brown dwarfs, most detailed white dwarf studies involve age calibration based on stellar cluster membership (Williams et al. 2004), and those with reliable ages rarely have simultaneous dynamical mass estimates (Girard et al. 2000; Bond 2009). HD 114174 thus represents a convenient system for studying the intricacies of post-main-sequence stellar evolution at low mass.

Without spectra, we cannot determine the chemical composition of HD 114174 B. Instead, we proceed by considering two different canonical cases: pure hydrogen and pure helium white dwarf atmospheres. By fitting theoretical models to our $J$ and $K$ observations, we can constrain the effective temperature, mass, and cooling age of HD $114174 \mathrm{~B}$ under reasonable assumptions about its composition.

\subsection{Synthetic Photometry and Fitting}

For pure hydrogen atmospheres and synthetic spectra, we use the theoretical models of Tremblay et al. (2011). ${ }^{11}$ The models include improved calculations for Stark broadening of hydrogen lines (Tremblay \& Bergeron 2009) and non-ideal perturbations, described within the occupation probability formalism of Hummer \& Mihalas (1988), directly inside the line profile calculations. The models also include Ly $\alpha$ profile calculations from Kowalski \& Saumon (2006). Convective energy transport is taken into account following the revised ML2 $/ \alpha=0.8$ prescription of mixing-length theory from Tremblay et al. (2010). Details regarding our helium atmosphere models are provided in Bergeron et al. (2011). Model grids cover a range in effective temperature between $T_{\text {eff }}=1500 \mathrm{~K}$ and $40,000 \mathrm{~K}$ in steps varying from $500 \mathrm{~K}$ to $5000 \mathrm{~K}$. The surface gravity ranges from $7.0 \leqslant \log g \leqslant 9.0$ by steps of $0.5 \mathrm{dex}$. Synthetic colors are obtained using the procedure of Holberg \& Bergeron (2006) based on Vega fluxes taken from Bohlin \& Gilliland (2004).

The method used to fit photometric data is similar to that described in Bergeron et al. (2001), which we briefly summarize. We first transform the magnitudes in each bandpass into observed average fluxes $f_{\lambda, m}$ using the following equation:

$$
m=-2.5 \log f_{\lambda, m}+c_{m}
$$

where

$$
f_{\lambda, m}=\frac{\int_{0}^{\infty} f_{\lambda} S_{m}(\lambda) d \lambda}{\int_{0}^{\infty} S_{m}(\lambda) d \lambda} .
$$

The transmission functions $S_{m}(\lambda)$ along with the constants $c_{m}$ for each bandpass are described at length in Holberg \& Bergeron (2006) and references therein. To make use of all photometric measurements simultaneously, we convert measured magnitudes into observed fluxes using Equation (1) and compare the resulting energy distributions with those predicted from our model atmosphere calculations. Model fluxes are also averaged over filter bandpasses by substituting $f_{\lambda}$ in Equation (2) for the monochromatic Eddington flux $H_{\lambda}$. The average observed

\footnotetext{
11 http://www.astro.umontreal.ca/ bergeron/CoolingModels/
}

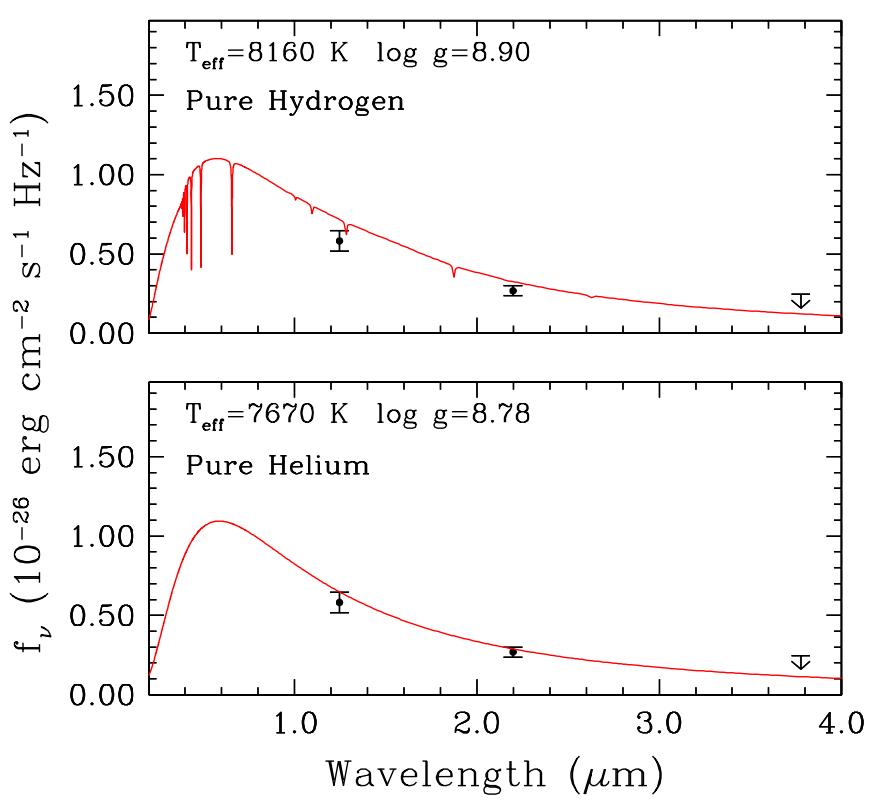

Figure 5. White dwarf model fits to our $J K L^{\prime}$ photometry assuming pure hydrogen (top) and pure helium (bottom) atmospheres. The observed $J K$ fluxes are represented by error bars. Model monochromatic fluxes are shown as the solid red line. Our measurements yield physical parameter estimates as listed in Table 2. The $L^{\prime}$-band non-detection from Keck is shown as an upper-limit.

(A color version of this figure is available in the online journal.)

fluxes $f_{\lambda, m}$ and model fluxes $H_{\lambda, m}$, which depend on $T_{\text {eff }}, \log g$ and $N(\mathrm{He}) / N(\mathrm{H})$, are related by

$$
f_{\lambda, m}=4 \pi(R / d)^{2} H_{\lambda, m}
$$

where $R / d$ is the ratio of the radius of the star to its distance from Earth.

Our fitting procedure relies on the nonlinear, least-squares, steepest descent method of Levenberg-Marquardt. The value of $\chi^{2}$ is taken as the sum over all bandpasses of the difference between both sides of Equation (3), properly weighted by the corresponding observational uncertainties. In our fitting procedure, we consider only $T_{\text {eff }}$ and the solid angle, $R / d$, as free parameters. We begin by guessing a value for the surface gravity $(\log g=8)$ and determine the corresponding effective temperature and solid angle. Combined with the distance, $d$, obtained from the trigonometric parallax measurement, this yields directly the radius of the star $R$. The radius is then converted into mass using evolutionary models similar to those described in Fontaine et al. (2001) but with C/O cores, $q(\mathrm{He}) \equiv \log M_{\mathrm{He}} / M_{\star}=10^{-2}$ and $q(H)=10^{-4}$, which are representative of hydrogen atmosphere white dwarfs, and $q(\mathrm{He})=10^{-2}$ and $q(H)=10^{-10}$, which are representative of helium atmosphere white dwarfs. In general, the $\log g$ value obtained from the inferred mass and radius $\left(g=G M / R^{2}\right)$ will be different from our initial guess of $\log g=8$, and the fitting procedure is thus repeated until an internal consistency in $\log g$ is reached. Figure 5 shows our white dwarf model fits to the photometric data.

Assuming a hydrogen-dominated atmosphere, we find: $T_{\text {eff }}=$ $8200 \pm 4000 \mathrm{~K}$ and $\log g=8.90 \pm 0.02$, which corresponds to a cooling age of $t_{c} \approx 3.4 \mathrm{Gyr}$ and model-dependent mass $m_{\text {model }}=1.15 \pm 0.01 M_{\odot}$. Assuming a helium-dominated atmosphere, we find: $T_{\text {eff }}=7700 \pm 4200 \mathrm{~K}, \log g=8.78 \pm 0.02$, $t_{c} \approx 3.5 \mathrm{Gyr}$, and $m_{\text {model }}=1.08 \pm 0.01 M_{\odot}$. With negligible differences between the physical parameter determinations 
when considering both models, we adopt the pure-H model values (Table 2 ). An $m=1.1 M_{\odot}$ remnant corresponds to an $\approx 6.2 M_{\odot}-6.9 M_{\odot}$ progenitor mass (Kalirai et al. 2008; Williams et al. 2009). Thus, HD 114174 B spent only a short period of time $(\approx 50 \mathrm{Myr})$ on the main-sequence.

\subsection{Period Range from Dynamics and Estimated Companion Mass}

With an estimate of the mass of HD 114174 B, we can constrain the system orbital period using dynamics. We first determine the true (physical) separation of HD 114174 B from HD 114174 A using the instantaneous Doppler acceleration $\left(57.4 \pm 0.4 \mathrm{~m} \mathrm{~s}^{-1} \mathrm{yr}^{-1}\right)$ and measured angular separation (692.1 \pm 8.7 mas) from geometry (e.g., Howard et al. 2010). We find that HD $114174 \mathrm{~B}$ is presently $59.8 \pm 0.4$ AU from its host star, much further than the projected separation $(18.09 \pm$ $0.34 \mathrm{AU})$. The true anomaly cannot be determined from the current data set. However, assuming the orbit configuration is near apastron or periastron, we can constrain the semimajor axis and thus orbital period. We find that the period is between 154 and $881 \mathrm{yr}$ for eccentricities in the range $0 \leqslant e \leqslant 0.5$. The period lower-limit corresponds to an expected RV semiamplitude of $K \approx 4400 \mathrm{~m} \mathrm{~s}^{-1}$ assuming an edge-on orbit, consistent with Figure 1.

Our numerical simulations indicate that $\approx 5 \%-10 \%$ of an orbit cycle is nominally required following a direct imaging discovery to place interesting constraints on all six orbit elements and the companion mass using dynamics for astrometric and Doppler RV signal-to-noise levels comparable to those of HD $114174 \mathrm{~B}$ (Crepp et al. 2012c). Thus, we may be able to determine the system orbit and mass of HD $114174 \mathrm{~B}$ as early as several years from now with sufficient observing cadence.

\section{SUMMARY AND DISCUSSION}

We report the discovery of a faint companion orbiting the nearby G5 star HD 114174. Initially identified as a promising high-contrast imaging target due to a measured long-term RV acceleration, we have used NIRC2 at Keck to obtain direct images of the source that is responsible for causing the Doppler trend. Multi-epoch detections in two different filters demonstrate association through common proper-motion over an $1.4 \mathrm{yr}$ time frame. The RV data spans $16.0 \mathrm{yr}$ and shows curvature (a change in the acceleration). Since HD 114174 has a precise parallax measurement, it will be possible to place strong constraints on the companion orbit (all six parameters) and mass as soon as the direct imaging astrometry shows curvature.

Our broadband photometric measurements indicate that the companion has neutral, or possibly blue colors $(J-K=$ $0.12 \pm 0.16$ ) suggesting interesting atmospheric physics. Deep ADI observations in the $L^{\prime}$-band under good seeing conditions at Keck resulted in a non-detection, further supporting the interpretation of a decreasing NIR spectral-energy distribution with increasing wavelength. HD $114174 \mathrm{~B}$ has an absolute magnitude $\left(M_{J}=13.97 \pm 0.11\right)$ and colors consistent with a T3-dwarf. However, dynamical considerations preclude the interpretation of a substellar companion. We calculate a firm minimum mass of $m>0.260 \pm 0.010 M_{\odot}$ using presently available RV and imaging data.

HD 114174 B appears to be a white dwarf. Using theoretical models to fit our photometry and assuming a pure hydrogen atmosphere, we estimate $T_{\text {eff }}=8200 \pm 4000 \mathrm{~K}$ and $\log g=$ $8.90 \pm 0.02$, which corresponds to a cooling age of $t_{c} \approx$
3.4 Gyr and remnant mass $m_{\text {model }}=1.15 \pm 0.01 M_{\odot}$, consistent with the $4.7_{-2.6}^{+2.3} \mathrm{Gyr}$ age estimate of the system based on isochronal analysis of HD $114174 \mathrm{~A}$, and also consistent with the dynamical mass lower limit. Calculations assuming a heliumrich atmosphere yield similar results.

Uncertainty in $T_{\text {eff }}$ is presently limited by the precision of our $J$ and $K$ photometry and current wavelength coverage. Further, the NIR colors of cool white dwarfs are known to exhibit an inversion (from having red colors to blue colors as $T_{\text {eff }}$ decreases) as the result of collisionally-induced $\mathrm{H}_{2}$ absorption. Thus, there exists a degeneracy in $T_{\text {eff }}$ for $J-K \approx 0.0$, possibly complicating the interpretation of HD $114174 \mathrm{~B}$ 's spectrophotometric signal at the lowest temperatures currently allowed by our models. For example, the white dwarf companion Gliese $86 \mathrm{~B}(J-K=1.0 \pm 0.3)$ was initially characterized as a brown dwarf (Els et al. 2001; Mugrauer \& Neuhäuser 2005). Deeper $L^{\prime}$-band observations obtained with the Large Binocular Telescope (LBT; Skrutskie et al. 2010; Skemer et al. 2012) and direct NIR spectroscopy measurements using an integral-field unit (Hinkley et al. 2011) will allow us to refine HD 114174 B's physical properties. Furthermore, it may be possible to study HD 114174 B at shorter wavelengths using Hubble Space Telescope in a fashion similar to that of Barstow et al. (2005) who obtained a spectrum of Sirius B with STIS.

HD $114174 \mathrm{~A}$ is a marginally evolved Sun-like star located at $d=26.14 \pm 0.37$ pc. Thus, it may be possible to measure its radius directly using CHARA interferometry, placing tighter constraints on the primary star's mass and hence the system age and also secondary mass (Boyajian et al. 2009). A similar analysis has been performed for the benchmark brown dwarf HR 7672 B (Crepp et al. 2012a). In the case of a white dwarf, a more accurate mass and age will likewise constrain physical properties of the progenitor. The HD 114174 system thus represents a useful testbed for studying white dwarf initialto-final mass ratios at a level of detail comparable to Sirius and Procyon. Given the anticipated sensitivity of forth-coming high-contrast imaging programs that use "extreme" $\mathrm{AO}$, it is likely that additional benchmark white dwarf companions will be (accidentally) uncovered in the near future. A more complete census of Sirius-like systems may ultimately be undertaken with the Gaia space mission using precision astrometry.

We thank Sasha Hinkley for trading NIRC2 observing time that allowed us to acquire observations of HD $114174 \mathrm{~B}$ in a complementary bandpass, demonstrating its unusual colors, and Mike Liu for pointing out reference to the Gliese 86 system which shares a similar story as HD 114174 B for being a white dwarf initially characterized as a brown dwarf. The referee, John Subasavage, provided helpful comments that improved the clarity of our manuscript. This research has made use of the SIMBAD database, operated at CDS, Strasbourg, France. The TRENDS high-contrast imaging program is supported by NASA Origins grant NNX13AB03G. J.A.J. is supported by generous grants from the David and Lucile Packard Foundation and the Alfred P. Sloan Foundation.

Data presented herein were obtained at the W. M. Keck Observatory, which is operated as a scientific partnership among the California Institute of Technology, the University of California and the National Aeronautics and Space Administration. The Observatory was made possible by the generous financial support of the W. M. Keck Foundation. The Center for Exoplanets and Habitable Worlds is supported 
by the Pennsylvania State University, the Eberly College of Science, and the Pennsylvania Space Grant Consortium.

\section{REFERENCES}

Barstow, M. A., Bond, H. E., Holberg, J. B., et al. 2005, MNRAS, 362, 1134 Bergeron, P., Leggett, S. K., \& Ruiz, M. T. 2001, ApJS, 133, 413

Bergeron, P., Wesemael, F., Dufour, P., et al. 2011, ApJ, 737, 28

Bohlin, R. C., \& Gilliland, R. L. 2004, AJ, 127, 3508

Bond, H. E. 2009, JPhCS, 172, 012029

Borysow, A., \& Frommhold, L. 1990, ApJL, 348, L41

Boyajian, T. S., McAlister, H. A., Cantrell, J. R., et al. 2009, ApJ, 691, 1243

Breton, R. P., Rappaport, S. A., van Kerkwijk, M. H., \& Carter, J. A. 2012, ApJ, 748,115

Carpenter, J. M. 2001, AJ, 121, 2851

Crepp, J. R., \& Johnson, J. A. 2011, ApJ, 733, 126

Crepp, J. R., Johnson, J. A., Fischer, D. A., et al. 2012a, ApJ, 751, 97

Crepp, J. R., Johnson, J. A., Howard, A. W., et al. 2012b, ApJ, 761, 39

Crepp, J. R., Johnson, J. A., Howard, A. W., et al. 2013, ApJ, 771, 46

Crepp, J. R., Johnson, J. A., \& Planet Search, C. 2012c, in American Astronomical Society Meeting Abstracts 220, Vol. 220 of American Astronomical Society Meeting Abstracts, 120.09

Crepp, J. R., Pueyo, L., Brenner, D., et al. 2011, ApJ, 729, 132

Crepp, J., Serabyn, E., Carson, J., Ge, J., \& Kravchenko, I. 2010, ApJ, 715,1533

Currie, T., Thalmann, C., Matsumura, S., et al. 2011, ApJL, 736, L33

Cushing, M. C., Rayner, J. T., \& Vacca, W. D. 2005, ApJ, 623, 1115

Els, S. G., Sterzik, M. F., Marchis, F., et al. 2001, A\&A, 370, L1

Fontaine, G., Brassard, P., \& Bergeron, P. 2001, PASP, 113, 409

Ghez, A. M., Salim, S., Weinberg, N. N., et al. 2008, ApJ, 689, 1044

Girard, T. M., Wu, H., Lee, J. T., et al. 2000, AJ, 119, 2428

Hinkley, S., Oppenheimer, B. R., Zimmerman, N., et al. 2011, PASP, 123, 74

Holberg, J. B. 2009, JPhCS, 172, 012022

Holberg, J. B., Barstow, M. A., Bruhweiler, F. C., Cruise, A. M., \& Penny, A. J. 1998, ApJ, 497, 935

Holberg, J. B., \& Bergeron, P. 2006, AJ, 132, 1221

Howard, A. W., Johnson, J. A., Marcy, G. W., et al. 2010, ApJ, 721, 1467

Hummer, D. G., \& Mihalas, D. 1988, ApJ, 331, 794

Kalas, P., Graham, J. R., Chiang, E., et al. 2008, Sci, 322, 1345
Kalirai, J. S., Hansen, B. M. S., Kelson, D. D., et al. 2008, ApJ, 676, 594

Kowalski, P. M., \& Saumon, D. 2006, ApJL, 651, L137

Lafrenière, D., Doyon, R., Marois, C., et al. 2007, ApJ, 670, 1367

Leggett, S. K., Burningham, B., Saumon, D., et al. 2010, ApJ, 710, 1627

Liebert, J., Young, P. A., Arnett, D., Holberg, J. B., \& Williams, K. A. 2005, ApJL, 630, L69

Liu, M. C., Fischer, D. A., Graham, J. R., et al. 2002, ApJ, 571, 519

Marcy, G. W., \& Butler, R. P. 1992, PASP, 104, 270

Marois, C., Lafrenière, D., Doyon, R., Macintosh, B., \& Nadeau, D. 2006, ApJ, 641,556

Marois, C., Macintosh, B., \& Véran, J.-P. 2010, Proc. SPIE, 7736, 77361J

Mugrauer, M., \& Neuhäuser, R. 2005, MNRAS, 361, L15

Muirhead, P. S., Vanderburg, A., Shporer, A., et al. 2013, ApJ, 767, 111

Oppenheimer, B. R., Hambly, N. C., Digby, A. P., Hodgkin, S. T., \& Saumon, D. 2001, Sci, 292, 698

Pueyo, L., Crepp, J. R., Vasisht, G., et al. 2012, ApJS, 199, 6

Quanz, S. P., Crepp, J. R., Janson, M., et al. 2012, ApJ, 754, 127

Skemer, A. J., Hinz, P. M., Esposito, S., et al. 2012, ApJ, 753, 14

Skrutskie, M. F., Cutri, R. M., Stiening, R., et al. 2006, AJ, 131, 1163

Skrutskie, M. F., Jones, T., Hinz, P., et al. 2010, Proc. SPIE, 7735, 77353H

Soummer, R., Pueyo, L., \& Larkin, J. 2012, ApJL, 755, L28

Tokovinin, A. 2004, in Revista Mexicana de Astronomia y Astrofisica Conference Series, ed. C. Allen \& C. Scarfe, Vol. 21 of Revista Mexicana de Astronomia y Astrofisica Conference Series, 7

Tokunaga, A. T., Simons, D. A., \& Vacca, W. D. 2002, PASP, 114, 180

Torres, G. 1999, PASP, 111, 169

Tremblay, P.-E., \& Bergeron, P. 2009, ApJ, 696, 1755

Tremblay, P.-E., Bergeron, P., \& Gianninas, A. 2011, ApJ, 730, 128

Tremblay, P.-E., Bergeron, P., Kalirai, J. S., \& Gianninas, A. 2010, ApJ, 712,1345

Valenti, J. A., \& Fischer, D. A. 2005, ApJS, 159, 141

Valenti, J. A., \& Piskunov, N. 1996, A\&AS, 118, 595

van Leeuwen, F. 2007, A\&A, 474, 653

Vogt, S. S. 1987, PASP, 99, 1214

Vogt, S. S., Allen, S. L., Bigelow, B. C., et al. 1994, Proc. SPIE, 2198, 362

Williams, K. A., Bolte, M., \& Koester, D. 2004, ApJL, 615, L49

Williams, K. A., Bolte, M., \& Koester, D. 2009, ApJ, 693, 355

Wizinowich, P., Acton, D. S., Shelton, C., et al. 2000, PASP, 112, 315

Wright, J. T. 2005, PASP, 117, 657

Zurlo, A., Vigan, A., Hagelberg, J., et al. 2013, A\&A, 554, 21 